\title{
Search for rare and lepton-flavour-violating decays of the Higgs boson with the ATLAS detector
}

\author{
Hanna Maria Borecka-Bielska* for the ATLAS Collaboration \\ University of Liverpool, United Kingdom \\ E-mail: hanna.maria.borecka@cern.ch
}

Searches for the decays of the Standard Model Higgs boson to dielectrons and to dimuons represent an important test of the yet unobserved Higgs boson couplings to first- and second-generation fermions. In addition, by looking for the Higgs boson lepton-flavour-violating processes such as $H \rightarrow e \mu$ or $H \rightarrow e \tau$ and $H \rightarrow \mu \tau$, which are forbidden in the Standard Model, it is possible to probe Beyond Standard Model theories allowing for such decays. The results of the presented searches are based on the partial and full Run-2 dataset, corresponding to an integrated luminosity of $36.1 \mathrm{fb}^{-1}$ and $139.0 \mathrm{fb}^{-1}$, respectively, collected by the ATLAS detector in $p p$ collisions at the LHC with the centre-of-mass energy of $13 \mathrm{TeV}$.

40th International Conference on High Energy physics - ICHEP2020

July 28 - August 6, 2020

Prague, Czech Republic (virtual meeting)

${ }^{*}$ Speaker 


\section{Introduction}

After the discovery of the Higgs boson in 2012 announced by the ATLAS [1] and CMS [2] experiments at CERN the era of precise measurements of its properties began. Searches for rare decays of the Higgs boson such as $H \rightarrow \mu \mu$ or $H \rightarrow e e$, and for lepton-flavour-violating (LFV) decays such as $H \rightarrow e \mu, H \rightarrow e \tau$ and $H \rightarrow \mu \tau$, are important in order to understand the Yukawa interactions in the Standard Model (SM) and to look for possible new physics contributions that may enhance these processes. The following sections present the recent results of searches for the above-mentioned Higgs boson decays using partial [3] and full Run-2 data [4, 5] collected by the ATLAS detector [6] during $p p$ collisions at the LHC.

\section{2. $H \rightarrow \mu \mu$}

The $H \rightarrow \mu \mu$ process is the most promising decay channel for the first observation of the Higgs boson coupling to second-generation fermions. However, searching for this decay is very challenging. It is characterised by a very low SM branching ratio (BR) of $2.17 \cdot 10^{-4}$ [7]. The search is dominated by large irreducible background composed of Drell-Yan (DY) dimuon pair production, diboson processes and processes with top quark, with the first one constituting over $90 \%$ as presented in Figure 1. The inclusive signal-to-background ratio $(S / B)$ in the mass region $m_{\mu \mu} \in(120,130) \mathrm{GeV}$, where the most signal is expected, is around $0.2 \%$. The analysis is divided

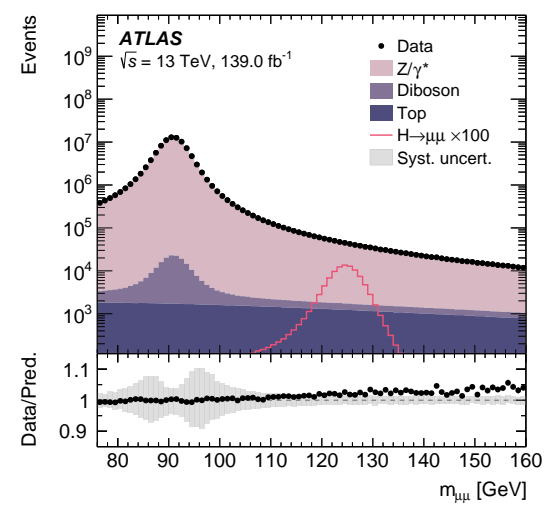

Figure 1: Dimuon invariant mass distribution in the inclusive category [4].

into several steps. First, events are required to pass a preselection, which is loose to maximise the signal acceptance. Then, they are divided into categories targeting different Higgs boson production modes. Four production modes are considered: gluon-gluon fusion $(g g \mathrm{~F})$, vector boson fusion (VBF) and the production associated with a vector boson $(V H)$ or a $t \bar{t}$ pair $(t \bar{t} H)$. To improve the dimuon mass resolution in categories with the highest production cross-section ( $g g \mathrm{~F}$ and $\mathrm{VBF}$ ), up to one final-state radiation photon is added to the mass calculation (this improves the resolution by $3 \%$ ). Finally, the signal is extracted from a signal+background $(S+B)$ fit to the dimuon mass distribution in each category separately.

A single $t \bar{t} H$ category is defined to target semi- or dileptonic decays of the $t \bar{t}$ pair and therefore the considered events are required to have at least one additional lepton $(e$ or $\mu)$ and at least one $b$-jet. 
The selection to the category is based on the output of a dedicated BDT classifier. The background is dominated by the $t \bar{t} Z$ and $t \bar{t}$ processes. The $V H$ process is targeted using two BDT classifiers, separately for events with one additional lepton ( $e$ or $\mu$ ) from the $W$ boson decay (3-lepton channel), and one for events with an additional pair of opposite-sign leptons from the $Z$ boson decay (4-lepton channel). Events with a $b$-jet are rejected. Two and a single category are defined in the 3- and 4-lepton channel, respectively. Background in these categories is dominated by diboson processes. Events failing selection for the $t \bar{t} H$ or $V H$ categories and having exactly one pair of opposite-sign muons and no $b$-jets are considered as coming from VBF or $g g F$ processes. These events are first divided according to the jet multiplicity into three $N$-jet channels: 0 -, 1- and 2-jet (which includes events with two or more jets), where dedicated BDT classifiers are trained. The separation into $N$-jet channels allows to use all possible information about the topology and kinematics of the process. It also separates the VBF events, which can be categorised with a special classifier in 2-jet channel taking into account the typical signature of this production process (presence of two forward jets with large rapidity gap between them). The VBF BDT classifier output divides events in 2-jet channel into four VBF-enriched categories. 2-jet events failing this selection are categorised into four $g g \mathrm{~F}$ categories. In the 1 - and 0 -jet channels events are split into four categories each based on the $g g$ F classifier score. The complete categorisation with 20 categories in total successfully separates different Higgs boson production modes. The expected $S / B$ varies in categories between $0.1 \%$ ad $20 \%$ and every category contributes to the final sensitivity between 0.1 and $0.6 \sigma$.

A $S+B$ fit is performed in each category separately to extract the signal as a narrow peak on top of a falling background. The signal distribution is modelled with a modified Crystal-Ball function to include power-law tails on both sides. Taking into account the very low inclusive $S / B$, it is essential to constrain the background at a per mill level. To address this, the background model is defined as a product of two functions. A core function is a LO DY line-shape [8] convolved with a Gaussian function to include resolution effects. It is a fully rigid function shared across all categories. To model backgrounds other than DY and to take into account any distortions due to selection and categorisation the core is multiplied by an empirical function. This function is selected from two families of functions (power-law functions or exponentials of polynomials) for each category individually based on a number of criteria and it is characterised by a certain number of free parameters.

The results show an observed signal yield with a significance of $2.0 \sigma$. The left side of Figure 2 presents the combined $S+B$ likelihood fit to weighted data. The combined observed signal strength, defined as a ratio of the observed to expected signal yield, is $1.2 \pm 0.6$ and is presented graphically on the right side of Figure 2, along with the signal strengths measured in five major groups of categories. The dominant uncertainties affecting the signal strength extraction are: data statistics $( \pm 0.58)$, signal theory systematics $\left({ }_{-0.08}^{+0.13}\right)$, signal experimental systematics $\left({ }_{-0.03}^{+0.07}\right)$ and the background modelling $( \pm 0.10)$.

\section{3. $H \rightarrow e e$ and $H \rightarrow e \mu$}

The SM branching ratio for the $H \rightarrow e e$ decay of $0.5 \cdot 10^{-9}$ is far below the sensitivity of any currently operating experiment. However, searching for this process is an important test of the SM as any hint of a signal would indicate that the Higgs mechanism does not work as expected. 

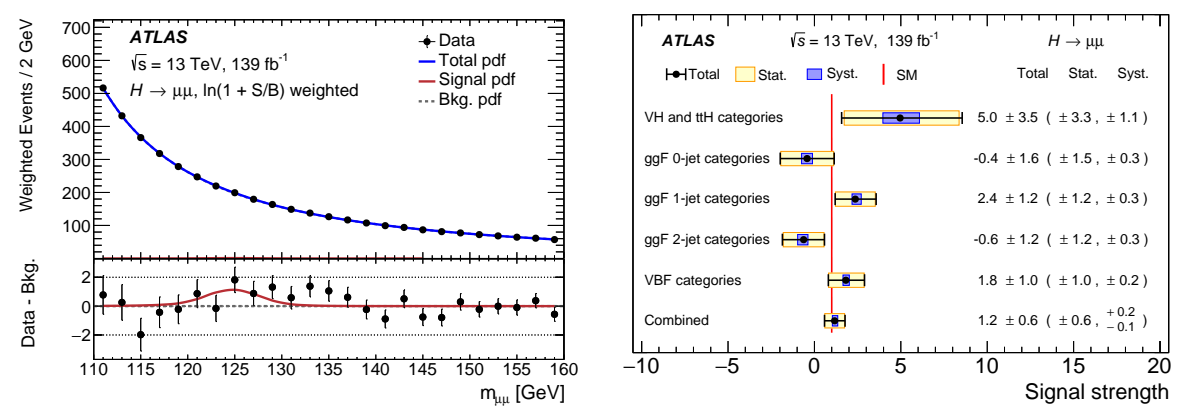

Figure 2: Inclusive $S+B$ likelihood fit to weighted dimuon mass distribution (left) and the observed signal strength in five major groups of categories along with the combined signal strength presented graphically (right) [4].

Additionally, LFV decays forbidden in the SM are allowed in a number of BSM theories. Both searches follow a strategy similar to the one used in the $H \rightarrow \mu \mu$ search using partial Run-2 data [9]. Events passing a loose dilepton selection are divided into cut-based categories targeting the VBF and $g g$ F Higgs boson production processes. The signal is extracted from a $S+B$ fit to the dilepton mass distribution in categories.

A single VBF category is defined by requiring a presence of two high- $p_{\mathrm{T}}$ jets, separated by $\Delta \eta\left(j_{1}, j_{2}\right)=3$ and with a high dijet mass, typical for VBF topology. Remaining events are divided into six $g g \mathrm{~F}$ categories, first according to dilepton $p_{\mathrm{T}}$ into High-, Medium- and Low- $p_{\mathrm{T}}^{\ell \ell}$ and then each of them is further split into Central and Forward categories based on the pseudorapidity of the leptons. In the $e \mu$ channel, there is an additional category for events with a low- $p_{\mathrm{T}}$ lepton, where the most fakes are expected.

In both searches, the signal is modelled as a sum of a Crystal Ball and a Gaussian function, however, due to a different composition of background processes, the background models differ. In the $H \rightarrow e e$ search, the background is composed of Drell-Yan, diboson and top processes. The $Z$ boson peak is described with a non-relativistic Breit-Wigner function convolved with a Gaussian function to account for resolution effects. It is summed with an exponential function divided by a cubic function to model the remaining backgrounds. In the $H \rightarrow e \mu$ channel, however, the background is smaller and composed from $Z / \gamma^{*} \rightarrow \tau \tau$, diboson, $W+$ jets processes and events with misidentified jets and was found to be well modelled with a Bernstein polynomial of degree two.

The results using full Run-2 data show no evidence for signal in either channel. The combined $S+B$ fits are presented in Figure 3 on the left for $H \rightarrow e e$ channel and on the right for $H \rightarrow e \mu$ decay. The best-fit value of the BR in the ee channel is $(0.0 \pm 1.7$ (stat. $) \pm 0.6$ (syst. $)) \cdot 10^{-4}$, with the observed (expected) upper limit at $95 \% \mathrm{CL}$ of $3.6 \cdot 10^{-4}\left(3.5 \cdot 10^{-4}\right)$. In the $e \mu$ channel the best-fit value of the BR is $(0.4 \pm 2.9$ (stat. $) \pm 0.3$ (syst. $)) \cdot 10^{-5}$. The observed (expected) upper limit at $95 \%$ $\mathrm{CL}$ is set at $6.2 \cdot 10^{-5}\left(5.9 \cdot 10^{-5}\right)$. In both analyses the data statistics is the dominant uncertainty and the largest systematic uncertainties are coming from the background modelling in the ee channel and Higgs boson production cross-section in the $e \mu$ channel. 

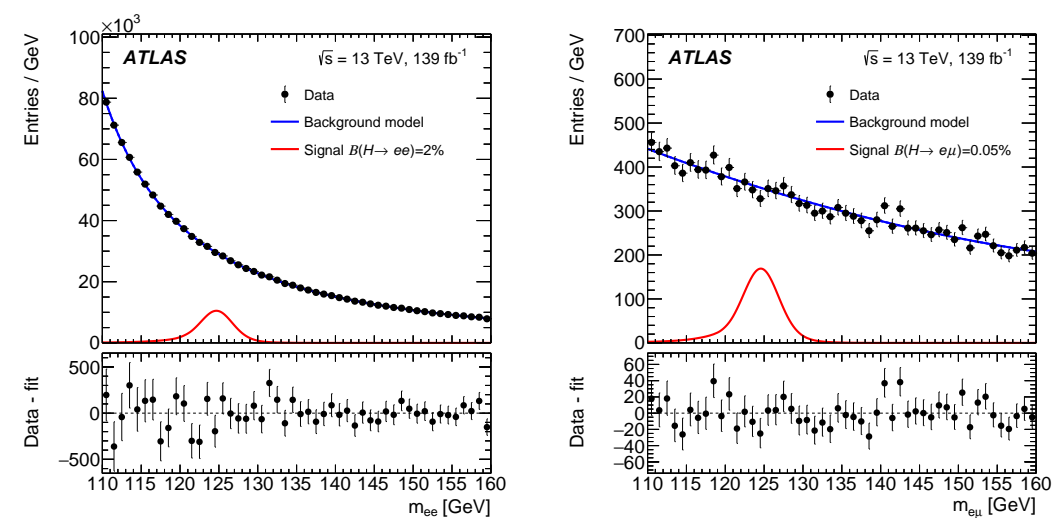

Figure 3: Combined $S+B$ likelihood fit to dilepton mass distribution in the $H \rightarrow e e$ (left) and $H \rightarrow e \mu$ channel (right) [5].

\section{4. $H \rightarrow e \tau$ and $H \rightarrow \mu \tau$}

The search for LFV Higgs boson decays with a $\tau$ in the final state is motivated by many new physics models that couple stronger to heavy third-generation leptons. In $H \rightarrow e \tau$ and $H \rightarrow \mu \tau$ analyses both leptonic and hadronic decays of the $\tau$ are considered. For the leptonic decays, only the ones with $\tau$ decaying to a lepton of different flavour than the primary lepton are considered to avoid large Drell-Yan background. As the reconstruction of invariant mass is non-trivial, the search is performed differently than $H \rightarrow e \mu$ analysis. Events passing a preselection are divided into VBF and non-VBF signal regions (SRs) using information about kinematics of the decays. Additionally, in case of leptonic decays of $\tau$, top and $Z \rightarrow \tau \tau$ control regions (CRs) are defined. BDT classifiers are trained to further separate signal from background in SRs. To constrain the background and extract branching ratios, a combined binned likelihood fit is performed to all BDT bins in SRs and the CRs yields independently in the $e \tau$ and $\mu \tau$ channels.

The background composition is much more complex than in the previously discussed searches. In both channels the $Z \rightarrow \tau \tau$ comprises the dominating background. Processes with top quarks are significant in channels with leptonic $\tau$ decays. The shape of these processes is obtained from Monte Carlo simulation and the normalisation from the fit. Background coming from events with misidentified objects is estimated with data-driven techniques. Backgrounds from other processes, such as $Z \rightarrow \mu \mu$, diboson, $H \rightarrow \tau \tau$ are constrained to the corresponding SM predictions.

Figure 4 shows example fits to BDT score in two SRs: $e \tau_{\mu}$ non-VBF on the left and $\mu \tau_{\text {had }}$ VBF on the right. The results show no evidence of signal in either channel. In the $e \tau$ channel the best-fit value of the BR is $0.15_{-0.17}^{+0.18} \%$ with the corresponding $95 \% \mathrm{CL}$ observed (expected) upper limit at $0.47 \%\left(0.34_{-0.10}^{+0.13} \%\right)$. The best-fit value of the BR in the $\mu \tau$ channel is $-0.22 \pm 0.19 \%$ and the observed (expected) limit is set at $0.28 \%\left(0.37_{-0.10}^{+0.14} \%\right)$. The dominating systematic uncertainties come from jet energy scale and estimation of background from misidentified objects.

\section{Summary}

Searches for rare and LFV decays are important as they allow to probe the Yukawa interactions in the SM. The presented results using data collected by the ATLAS detector in Run 2 show no 
significant evidence for a signal in any of the presented decay channels, although the $H \rightarrow \mu \mu$ search is approaching sensitivity to the SM Higgs boson rate. The upper limits have been set on the corresponding branching ratios and they are graphically presented in Figure 5.
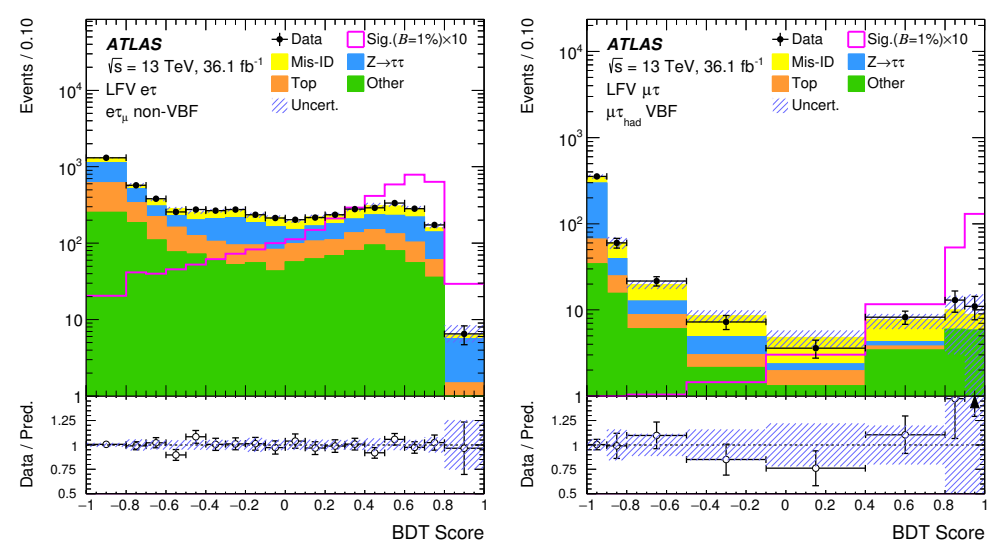

Figure 4: Example post-fit BDT score distribution in the $e \tau_{\mu}$ non-VBF (left) and $\mu \tau_{\text {had }}$ VBF (right) signal regions [3].

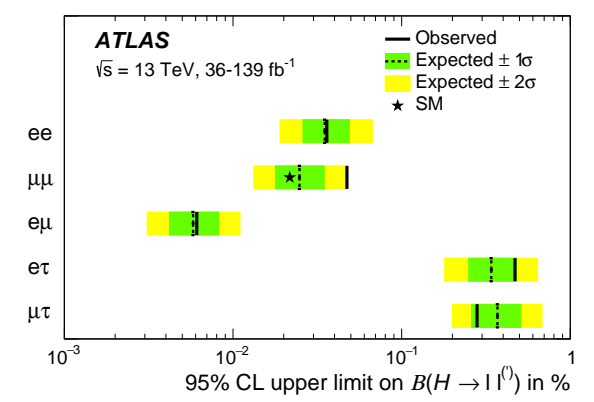

Figure 5: Upper limits at 95\% CL for the branching ratios of discussed decays [4].

\section{References}

[1] ATLAS Collaboration, Phys. Lett. B 716 (2012) 1

[2] CMS Collaboration, Phys. Lett. B 716 (2012) 30

[3] ATLAS Collaboration, Phys. Lett. B 800 (2020) 135069

[4] ATLAS Collaboration, arXiv: 2007.07830 [hep-ex]

[5] ATLAS Collaboration, Phys. Lett. B 801 (2020) 135148

[6] ATLAS Collaboration, JINST 3 (2008) S08003

[7] D. de Florian et al., arXiv: 1610.07922 [hep-ph]

[8] ATLAS Collaboration, JHEP 12 (2017) 059

[9] ATLAS Collaboration, Phys. Rev. Lett. 119 (2017) 051802 